\title{
Crystal plasticity modeling of irradiation effects on flow stress in pure-iron and iron-copper alloys
}

\author{
Pritam Chakraborty*, S. Bulent Biner* \\ Fuel Modeling and Simulation Department, Idaho National Laboratory, Idaho Falls, ID 83401, USA
}

\begin{abstract}
The mechanistic modeling of irradiation induced embrittlement of reactor pressure vessel steels strongly depends on the precise evaluation of flow stress behavior. This requires accurate characterization of change in both the yield strength as well as the strain-hardening capacity. A dislocation-density based crystal plasticity model is thus developed in this work to quantify these variations with irradiation. The model considers the interaction between dislocations and irradiation induced defects such as self-interstitial atomic loops, vacancy clusters and precipitates to obtain flow stress variations in irradiated ferritic alloys. The model is calibrated and validated for polycrystalline pure-iron and iron-copper alloys, neutron-irradiated to different dose levels under typical pressure vessel operating conditions. A comparison with experimental results show that the model is able to quantify the changes in flow stress behavior accurately. At 0.2 dpa a loss of strain-hardening capacity beyond $2 \%$ strain is also obtained from the model. The yield strength increase with irradiation obtained from the model is also compared with analytical strengthening models based on Orowan's equation.
\end{abstract}

\section{Introduction}

The long-term exposure of Reactor Pressure Vessels (RPVs) to neutron irradiation increase their failure probability due to the precipitation and segregation of secondary phases, as well as formation and growth of vacancy clusters and self interstitial atomic loops [1]. For instance, an increase in the yield strength and a decrease in the post-yield hardening exponent can be observed with increasing irradiation dose levels, and is due to the interaction of the dislocations with the defects. Also, the ductile-to-brittle transition temperature (DBTT) has been observed to increase with irradiation dose levels. Since, the RPV is one of the critical components whose integrity determines the safe extension of the operational lifetime of the exisiting nuclear reactors beyond the 60 year period, hence, accurate assessment of RPV steel embrittlement is essential.

The present approach to quantify embrittlement in RPVs rely on semi-empirical models that are calibrated using the surveillance samples in the reactors [2]. Though these models have successfully quantified DBTT shifts and yield stress increase for the 40-60 year life

\footnotetext{
${ }^{*}$ Corresponding author

Email address: pritam.chakraborty@inl.gov (Pritam Chakraborty)
} 
extension, their accuracy for extrapolation can be hindered due to the lack of experimental data and appearance of late-blooming phases. Multi-scale models of irradiation induced aging can assist in improving the predictive ability of the semi-emperical models by providing surrogate data, and, exploring the mechanisms that can emerge due to longer exposures beyond the designed period [3]. These models span across widely differing length and timescales from atomistics to engineering scale, and attempts to provide physical understanding of defect formation, their interaction and effect on mechanical behavior.

At the scale of the fracture specimens, the models developed to characterize DBTT shifts primarily incorporate the mechanisms causing stable ductile damage and probabilistic cleavage initiation. For ductile damage, pressure sensitive plasticity models characterizing void nucleation, growth and coalescence are utilized $[4,5]$. The model parameters are calibrated from tensile tests and/or fracture tests at the upper shelf. The cleavage initiation models are based on the weakest link theory, which incorporates the mechanism of plasticity induced micro-crack initiation from grain-boundary carbides and their growth into a macrocrack causing unstable failure. The Beremin model [6], using the weakest link theory based Weibull distribution [7] for probabilistic cleavage initiation, is typically employed to capture the scattered fracture energy and toughness observed in the transition regime. The Beremin model parameters are typically calibrated near the lower shelf.

A combination of the ductile damage and Beremin model has been used in Finite Element Method (FEM) simulations of fracture specimens to obtain DBTT by several authors [8-11]. In [10], the calibrated ductile damage and Beremin model parameters are kept constant and only the temperature sensitive flow stress is varied to obtain the DBTT of unirradiated RPV steels. In [8], temperature dependent Beremin model parameters are considered additionally to obtain improved comparison with experiments. Under irradiated condition it has been observed that both the ductile damage and cleavage model parameters vary with dose levels [9]. Also, the DBTT shifts are sensitive to the post-yield hardening behavior. In [11], temperature dependent cohesive zone model and flow stress parameters were utilized to obtain DBTT in RPV steels. In a parametric study using tensile test data for ferritic-martensitic steels [12], it has been shown that the variation of ductile damage parameters saturate with increasing irradiation dose levels, whereas the flow stress parameters still evolve. From these numerical studies it can be concluded that precise evaluation of flow stress variation with temperature and irradiation dose levels is essential to characterize DBTT shifts accurately. Also, quantifying the yield stress variations alone is not sufficient to obtain the DBTT shifts and evaluation of flow stress variations upto a critical plastic strain is necessary [13].

Modeling the evolution of flow stress with irradiation involves the development of methods to track defect evolution and their interaction with dislocations. Atomistic scale method such as Molecular Dynamics (MD) can provide insight into the fundamental mechanisms and the associated energetics. Coarse grained methods such as Atomistic Kinetic Monte Carlo (AKMC) and Dislocation Dynamics (DD), can subsequently utilize the information from MD to model larger spatial and temporal scales. Meso-scale methods such as phasefield, cluster dynamics and crystal plasticity can further extend the length and time scales of defect evolution and interaction to polycrystalline systems. The predictions from the crystal-plasticity models can then be utilized to obtain the flow stress variations at the specimen-scale simulations. Hence in the present work, a crystal plasticity based modeling approach is being developed to quantify the interaction between irradiation induced defects 
and dislocations with the objective to obtain correlations between irradiation dose levels and flow stress variations in RPV steels.

In the present work, pure-iron and iron-copper model alloy systems for RPV steels are considered to quantify the effect of neutron irradiation on flow stress variation. The experimental results presented in [14-16] are utilized for this purpose. In [14, 15], specimens made from a RPV steel and the model alloys were irradiated around $290{ }^{\circ} \mathrm{C}$ and $15 \mathrm{MPa}$ to a maximum dose-level of $0.1 \mathrm{dpa}$ followed by defect and flow stress characterization. To quantify the defect type, number density and size, different techniques such as Transmission Electron Microscopy (TEM), Positron Annihilation Spectroscopy (PAS), Atom Probe Tomography (APT) and Small Angle Neutron Scattering (SANS) were used. Flow stress variations were obtained from quasi-static tensile testing performed at room temperature. Furthermore, the analytical model based on Orowan strengthening was utilized to investigate the effect of different defect types on the yield stress [15]. From their analysis it was concluded that the self-interstitial atomic (SIA) loops, and, vacancy and copper clusters are the strongest obstacle to dislocation motion in pure-iron and iron-copper alloys. The contribution of very small vacancy clusters to overall hardening was insignificant. These observations were included in the crystal plasticity model where the strengthening effect of SIA loops, and vacancy and copper clusters was only considered. Subsequently, the parameters are calibrated from and validated with unirradiated and irradiated microstructures and flow stress data, to establish the workability of the model.

The organization of the paper is as follows. In section 2, the dislocation density based crystal plasticity model considering the self-interactions of dislocations and with irradiation induced defects, is presented. A comparison of the model predictions with dislocation dynamics simulation [17] is presented in sub-section 3.1. Subsequently, the model parameters are calibrated and validated for unirradiated and irradiated pure-iron and iron-copper alloys, and presented in sub-section 3.2. Finally in sub-section 3.3, the prediction of increase in yield strength from the crystal plasticity model is compared with an analytical model. The paper is concluded in section 4 .

\section{Dislocation-density based crystal plasticity model}

In the crystal plasticity model [18], the deformation gradient tensor, $\underline{\underline{F}}$, is multiplicatively split as

$$
\underline{\underline{F}}=\underline{\underline{F}}^{e} \underline{\underline{F}}^{p}
$$

where $\underline{\underline{F}}^{e}$ and $\underline{\underline{F}}^{p}$ are the elastic and plastic deformation gradients, respectively. The multiplicative split assumes an intermediate configuration where the inelastic deformation occurs through dislocation motion along specific slip systems. This configuration is subsequently stretched and rotated elastically to the current configuration by $\underline{\underline{F}}^{e}$. The rate of evolution of $\underline{\underline{F}}^{p}$ occurs through glide of dislocations along different slip systems as

$$
\underline{\underline{F}}^{p}=\sum_{\alpha} \dot{\gamma}^{\alpha} \underline{m}_{0}^{\alpha} \otimes \underline{n}_{0}^{\alpha}
$$


where $\dot{\gamma}^{\alpha}$ is the glide rate on a slip system, $\alpha$, and, $\underline{m}_{0}^{\alpha}$ and $\underline{n}_{0}^{\alpha}$ is the glide direction and plane normal, respectively, in the intermediate configuration. The glide rate on a slip system in Eq.2 evolves following the Orowan's equation

$$
\dot{\gamma}^{\alpha}=\rho_{M}^{\alpha} b^{\alpha} v^{\alpha}
$$

where $\rho_{M}^{\alpha}$ is the density of mobile dislocation, $b^{\alpha}$ is the Burger's vector and $v^{\alpha}$ is velocity of mobile dislocations on the slip system. In this model, the density of dislocations that has the potential to be thermally activated is identified as mobile. The converse holds true for the immobile dislocation density, as discussed in [19]. This is consistent with the observation that when the grains are initially deforming elastically, though none of the dislocations are moving, a finite density of dislocations have the potential to be mobile once they can overcome the barriers. These barriers could be intrinsic, such as the Peierls barrier, as well as extrinsic in the form of precipitates, parallel and forest dislocations [20]. The $v^{\alpha}$ is defined using the activation enthalpy driven flow rule [21] as

$$
v^{\alpha}= \begin{cases}l^{\alpha} \nu \exp \left(-\frac{\Delta F}{K_{B} T}\left(1-\left[\frac{\left|\tau^{\alpha}\right|-s_{a}^{\alpha}}{s_{t}^{\alpha}}\right]^{p}\right)^{q}\right) \operatorname{sgn}\left(\tau^{\alpha}\right), & \text { for }\left|\tau^{\alpha}\right|>s_{a}^{\alpha} \\ 0, & \text { otherwise }\end{cases}
$$

where $l^{\alpha}$ is the average dislocation glide distance between barriers, $\nu$ is the jump frequency, $\Delta F$ is the activation energy under zero stress, $K_{B}$ is the Boltzmann's constant, $T$ is the temperature in Kelvin, $\tau^{\alpha}$ is the resolved shear stress, and, $s_{a}^{\alpha}$ and $s_{t}^{\alpha}$ are the athermal and thermal resistances to slip, respectively. The $\tau^{\alpha}$ is related to the $2^{\text {nd }}$ Piola-Kirchhoff stress in the intermediate configuration $\left(\underline{\underline{T}}^{*}\right)$ following

$$
\tau^{\alpha}=\underline{\underline{T}}^{*}: \underline{m}_{0}^{\alpha} \otimes \underline{n}_{0}^{\alpha}
$$

The evolution of the athermal resistance, $s_{a}^{\alpha}$, is based on the dispersed barrier hardening model

$$
s_{a}^{\alpha}=\left(\left(s_{\rho-\rho}^{\alpha}\right)^{r}+\left(s_{\rho-S I A}^{\alpha}\right)^{r}+\left(s_{\rho-P P T}^{\alpha}\right)^{r}\right)^{\frac{1}{r}}
$$

and has contributions from dislocation-dislocation $\left(s_{\rho-\rho}^{\alpha}\right)$, dislocation-SIA loop $\left(s_{\rho-S I A}^{\alpha}\right)$ and dislocation-vacancy and copper clusters $\left(s_{\rho-P P T}^{\alpha}\right)$. In Eq.6, $r$ determines the superposition law ranging from linear $(r=1)$ to quadratic $(r=2)$. The thermal resistance, $s_{t}^{\alpha}$, depends on short-range obstacles and is related to the Peierl's barrier for BCC system [21].

The evolution of the mobile dislocation density, $\rho_{M}^{\alpha}$, follows [21]

$$
\rho_{M}^{\dot{\alpha}}=\frac{k_{m u l}}{b^{\alpha}} \sqrt{\sum_{\beta} \rho_{M}^{\beta}}\left|\dot{\gamma}^{\alpha}\right|-\frac{2 R_{c}}{b^{\alpha}} \rho_{M}^{\alpha}\left|\dot{\gamma}^{\alpha}\right|-\frac{1}{b^{\alpha} \lambda^{\alpha}}\left|\dot{\gamma}^{\alpha}\right|
$$

where the first term represents dislocation multiplication following Frank-Reed mechanism. The second term represents annihilation of dislocations of opposite signs, and, the third term represents the immobilization of dislocations due to trapping by dislocations and defects. In Eq.7, $k_{m u l}$ is the multiplication factor, $R_{c}$ is the self-annihilation radius of dislocations with opposite signs and $\lambda^{\alpha}$ is the mean trapping distance. The conversion from mobile to immobile dislocations in this model is based on [22] that considers this transformation 
through the effective mean free path of statistical trapping. A harmonic average of the trapping distances from different obstacles such as dislocations, voids and precipitates is incorporated as proposed in [23]. In the absence of any defect, the motion of dislocations can be obstructed by both parallel and forest dislocations. In this work, only the trapping by the inplane dislocations is accounted for following [21] using

$$
\frac{1}{\lambda^{\alpha}}=\beta_{\rho} \sqrt{\rho^{\alpha}}
$$

where $\beta_{\rho}$ is a scaling factor, and $\rho^{\alpha}=\rho_{M}^{\alpha}+\rho_{I}^{\alpha}$ is the total dislocation density. The evolution of the immobile dislocation density, $\rho_{I}^{\alpha}$, is defined as

$$
\dot{\rho}_{I}^{\alpha}=\frac{1}{b^{\alpha} \lambda^{\alpha}}\left|\dot{\gamma}^{\alpha}\right|-k_{d y n} \rho_{I}^{\alpha}\left|\dot{\gamma}^{\alpha}\right|
$$

where the second term represents the dynamic recovery of the immobile dislocations and $k_{d y n}$ is the dynamic recovery factor.

The contribution of dislocation-dislocation interaction to the athermal resistance is described following [24] as

$$
s_{\rho-\rho}^{\alpha}=q_{p} G b^{\alpha} \sqrt{\sum_{\beta} A^{\alpha \beta} \rho^{\beta}}
$$

where $G$ is the shear modulus, $A^{\alpha \beta}$ is the matrix with interaction coefficients of dislocations between different slip systems, and $q_{p}$ is the strength parameter.

The interaction between dislocations and the SIA loops is described using the model proposed in [25]. In this model a second order defect tensor is defined as

$$
\underline{\underline{H}}=\sum_{l} 3 d_{i}^{l} N_{i}^{l}\left(\underline{\underline{I}}-\underline{n}_{i}^{l} \otimes \underline{n}_{i}^{l}\right)
$$

where $d_{i}^{l}$ is the hexagonal SIA loop diameter, $N_{i}^{l}$ is the initial number density of loops and $n_{i}^{l}$ are the normals to the loop planes. This form of defect description allows the anisotropic interaction between the $1 / 2\langle 111\rangle$ and $\langle 100\rangle$ SIA loops and dislocations. For instance, the motion of mobile dislocations are unobstructed by loops whose habit planes coincide with the corresponding dislocation glide planes. Thus, the contribution to athermal resistance due to this interaction can be represented as

$$
s_{\rho-S I A}^{\alpha}=q_{i} G b^{\alpha} \sqrt{\underline{\underline{N^{\alpha}}}: \underline{\underline{H}}}
$$

where $\underline{\underline{N}}^{\alpha}=\underline{n}_{0}^{\alpha} \otimes \underline{n}_{0}^{\alpha}$ is a second order tensor of slip plane normals $\left(\underline{n}_{0}^{\alpha}\right)$ appearing in Eq.2. The SIA loops trap the dislocations, thus immobilizing them. This effect is incorporated through

$$
\frac{1}{\lambda^{\alpha}}=\beta_{\rho} \sqrt{\rho^{\alpha}}+\beta_{i} \sqrt{\underline{\underline{N^{\alpha}}}: \underline{\underline{H}}}
$$

where $\lambda^{\alpha}$ is the mean trapping distance (Eq.8) and $\beta_{i}$ is a scaling factor. However, once the dislocations have enough driving force to mobilize by overcoming the barriers, they annihilate the loops, which is defined by 


$$
\underline{\underline{\dot{H}}}=-\eta \sum_{\alpha}\left(\underline{\underline{N}}^{\alpha}: \underline{\underline{H}}\right) \underline{\underline{N}}^{\alpha}\left|\dot{\gamma}^{\alpha}\right|
$$

in [25], where $\eta$ is the scaling factor for annihilation. The pinning of mobile dislocations by the SIA loops and their subsequent annihilation leads to the formation of clear channels that has been observed experimentally [26]. The form of Eq.14 ensures that the loops are not annihilated by mobile dislocations whose slip planes coincides with the habit planes of the loop.

The resistance of vacancy and copper clusters to dislocation motion is incorporated following $[27]$ as

$$
s_{\rho-P P T}^{\alpha}=q_{c} G b^{\alpha} \ln \left(\frac{1}{2 b^{\alpha} \sqrt{N \times d}}\right) \sqrt{N \times d}
$$

where is $q_{c}$ the strength factor, and, $d$ and $N$ are the mean defect size and number density, respectively. In this expression it is assumed that the vacancy and copper clusters are randomly distributed and spherical in shape, thus providing an isotropic resistance to slip. Also, from MD simulations of copper precipitates with sizes ranging from 1-3 nm, it was observed that Orowan shearing is the dominant mechanism [28]. Based on their observation it is assumed in this work that the vacancy and copper clusters provides a similar resistance to dislocation motion via viscous drag accounted through Eq. 15 without increasing the density of immobile dislocations, which is typical of Orowan looping. Hence, the mean trapping distance $\left(\lambda^{\alpha}\right.$ in Eq.13) is not modified by these clusters.

\section{Results and Discussion}

The crystal plasticity model is implemented in the Grizzly code that is derived from MOOSE, a FEM based object oriented software aimed to solve strongly coupled multi-physics problem [29]. The rate equations appearing in the constitutive model are integrated using an implicit backward-Euler scheme following [30]. The details of the numerical implementation is provided in [31]. At first the workability of the model is verified against DD simulations reported in $[17,25]$ and detailed in sub-section 3.1. The calibration and validation of the model is then performed in sub-section 3.2 utilizing the experimental results provided in [14-16]. A sensitivity analysis of the flow stress to initial dislocation densities and grain orientation is also performed in this sub-section. Further, in sub-section 3.3, the yield stress values predicted from the crystal plasticity model is compared with the estimates made from the analytical model presented in [15].

\subsection{A comparitive study between crystal plasticity and dislocation dynamics models}

A DD model has been developed in [17] to investigate the interaction between the $1 / 2<$ $111>$ SIA loops and dislocations in bcc-iron. Simulations with varying loop densities were then performed that showed initial pinning of dislocations, followed by the annihilation of loops by the dislocations and clear channel formation. A crystal plasticity model was subsequently proposed in [25] to capture this localization event and has been adopted in this work. Though, the dislocation-SIA loop interaction laws are unmodified from [25] (Eqs.12, 
14), a more detailed dislocation evolution model is used in this work as compared to [25]. Hence, at first, the workability of the model is compared with the DD simulations.

In the crystal plasticity based FEM (CPFEM) simulations, a single element of size $1 \mu m \times$ $1 \mu m \times 1 \mu m$ is considered. The $\mathrm{X}, \mathrm{Y}$, and $\mathrm{Z}$ axes of the simulation domain is aligned with [1 0 0 0], [0 $0.99985-0.01745]$ and [ 00.01745 0.99985] of the crystal axes, respectively. The element is displaced along the Z-axis of the simulation domain with a strain rate of 100/s similar to [17]. Due to the small initial orientation of the single element, asymmetry in glide is observed. Initial mobile and immobile dislocation densities are assigned equal values of $10 \mu \mathrm{m}^{-2}$ based on the total density of $20 \mu \mathrm{m}^{-2}$ reported in [17]. DD simulation were performed in [17] considering different SIA loop densities and diameter of 100b (24.8 nm for bcc-iron). The same defect size (d) and density $(\mathrm{N})$ values are used to perform the CPFEM simulations. The stress-plastic strain curves for $\mathrm{N}=0$ and $\mathrm{N}=8.15 \times 10^{3} \mu \mathrm{m}^{3}$ are first utilized to calibrate the model parameters and are shown in Table 1. A linear superposition of the different athermal resistance components with $\mathrm{r}=1$ (Eq.6) is considered and is similar to [25]. The parameters $l^{\alpha}, \nu, \Delta F, p, q$ and $A^{\alpha \beta}$ are obtained from [21]. Subsequently, the stress-plastic strain curves obtained from CPFEM simulations for $\mathrm{N}=8.15 \times 10^{2} \mu \mathrm{m}^{3}$ and $\mathrm{N}=1.63 \times 10^{4} \mu \mathrm{m}^{3}$ are compared with DD simulations in Fig.1.

The evolution of mobile, immobile and defect densities is compared between the different cases in Fig.2. The maximum value of the mobile and immobile dislocation densities amongst the 48 slip systems are compared between the different cases in Fig.2(a) and 2(b), respectively. Almost similar evolution of the maximum mobile dislocation densities can be observed in Fig. 2(a) for all the cases. However, the evolution rate of immobile dislocation densities significantly increases with defect density as can be observed from Fig.2(b) and is due to the trapping of the mobile dislocations by the loops. Since, the evolution of mobile dislocations is similar for all the cases, an increase in the defect density increases their probability of annihilation and is evident from a larger decrease of the norm of the defect tensor in Fig.2(c). Overall, a satisfactory agreement with DD simulations can be obtained from the crystal plasticity model with this parameter set. 


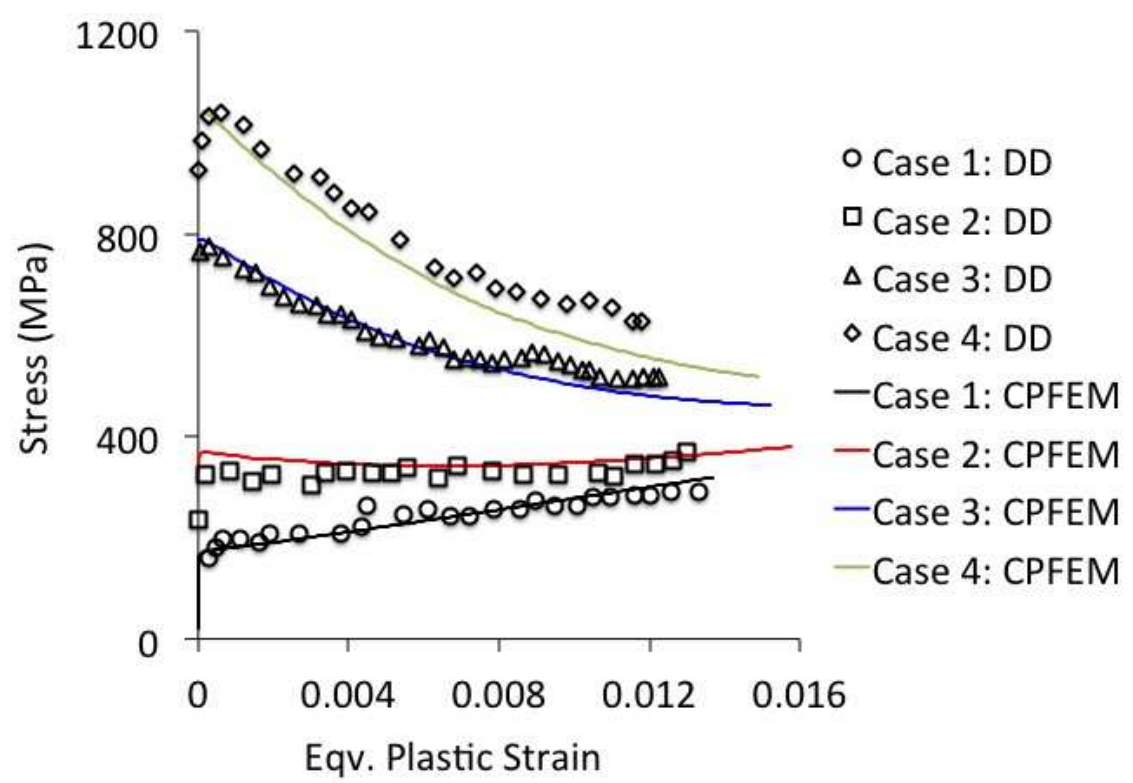

Figure 1: Comparison of stress-strain evolution between crystal plasticity finite element method (CPFEM) and dislocation dynamics (DD) simulations [17]. The different cases represent the SIA loop densities (N) as follows: Case 1 - 0; Case $2-8.15 \times 10^{2} \mu m^{3}$; Case $3-8.15 \times 10^{3} \mu m^{3}$; Case $4-1.63 \times 10^{4} \mu m^{3}$. In all the cases the same loop size of $24.8 \mathrm{~nm}$ is used consistent with [17]. The stress-plastic strain curve for DD is reproduced from [25]. 
Table 1: Parameters of crystal plasticity model calibrated from DD simulations

\begin{tabular}{|c|c|c|c|c|c|}
\hline$s_{t}^{\alpha}(\mathrm{MPa})$ & 50 & $k_{m u l}$ & 0.9 & $R_{c}(\mathrm{~nm})$ & 8 \\
\hline$k_{d y n}$ & 100 & $q_{p}$ & 0.2 & $q_{i}$ & 0.37 \\
\hline$\beta_{\rho}$ & 0.4 & $\beta_{i}$ & 0.1 & $\eta$ & 120 \\
\hline
\end{tabular}

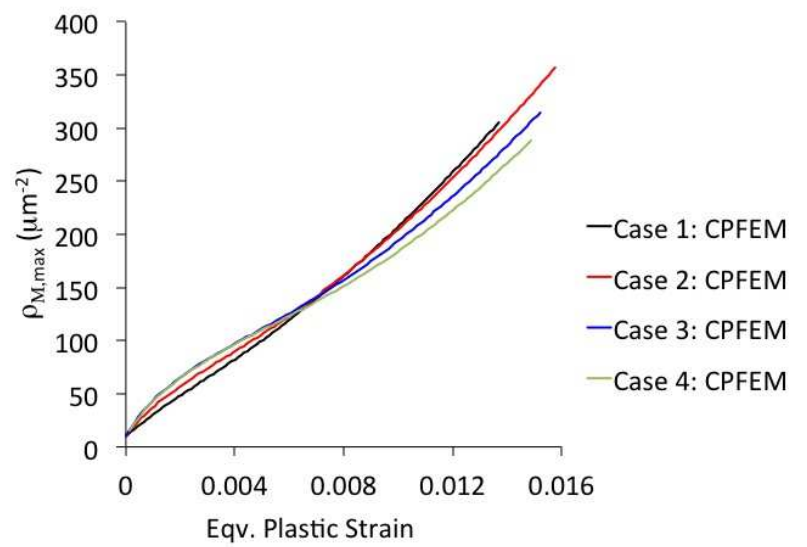

(a)

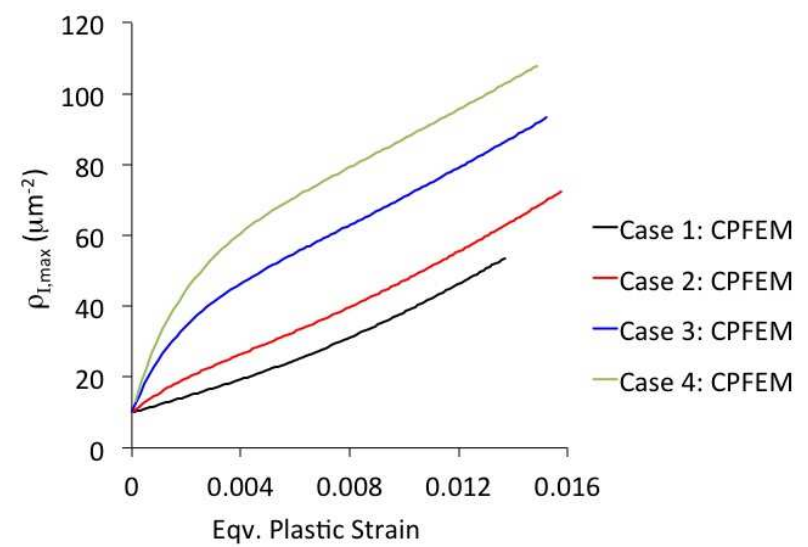

(b)

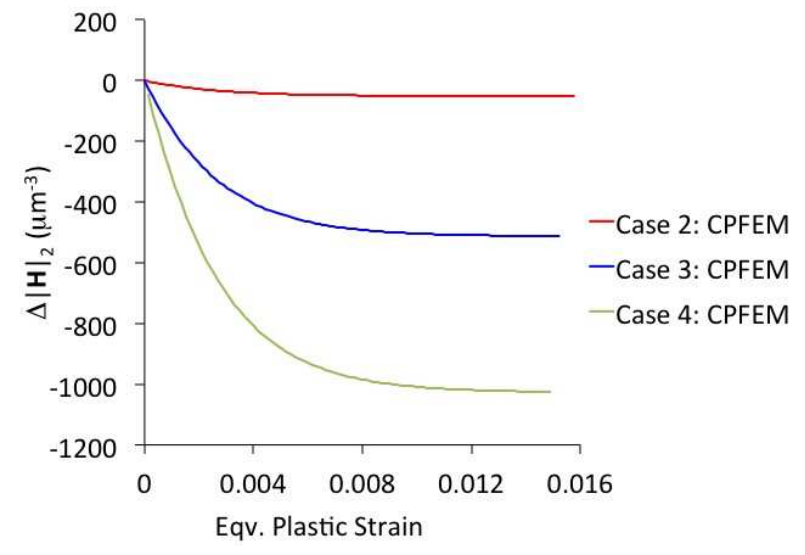

(c)

Figure 2: Comparison of (a) maximum mobile dislocation density, (b) maximum immobile dislocation density and (c) change in the $\mathrm{L}_{2}$-norm of defect tensor, between the different cases from the crystal plasticity model.

\subsection{Calibration and validation of the crystal plasticity model parameters for pure-iron and} iron-copper alloys

The stress-strain curves for the unirradiated and irradiated iron and iron-copper alloys provided in [14] are used to calibrate the model parameters. A representative volume of $500 \mu \mathrm{m} \times 500 \mu \mathrm{m} \times 500 \mu \mathrm{m}$ containing 64 cubic grains of size $125 \mu \mathrm{m} \times 125 \mu \mathrm{m} \times 125 \mu \mathrm{m}$ is considered for CPFEM simulations. Each of the grains is associated with a single element and a random orientation. Since, the objective of these simulations is to evaluate the average response of the representative volume, such an approximation significantly reduces the computational cost while maintaining the desired accuracy. Similar analyses have been 
performed in [32] to characterize the deformation behavior of Ti-6242 under constant strainrate and creep loading. The representative volume is fixed along $\mathrm{X}, \mathrm{Y}$ and $\mathrm{Z}$ directions on the back, left and bottom faces, respectively. The top face is displaced along the Z-direction with a strain rate of $10^{-4} / \mathrm{s}$ to obtain the uniaxial stress-strain response of the representative volume. The FE discretized volume with the spatial distribution of one of the Euler angles and the boundary conditions are shown in Fig.3(a) and 3(b), respectively.

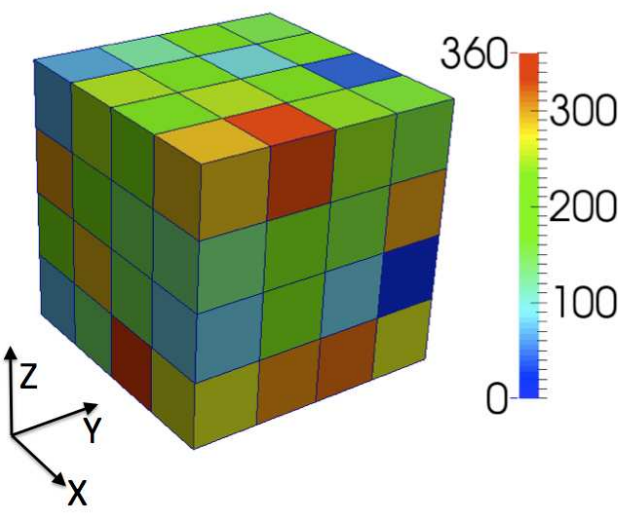

(a)

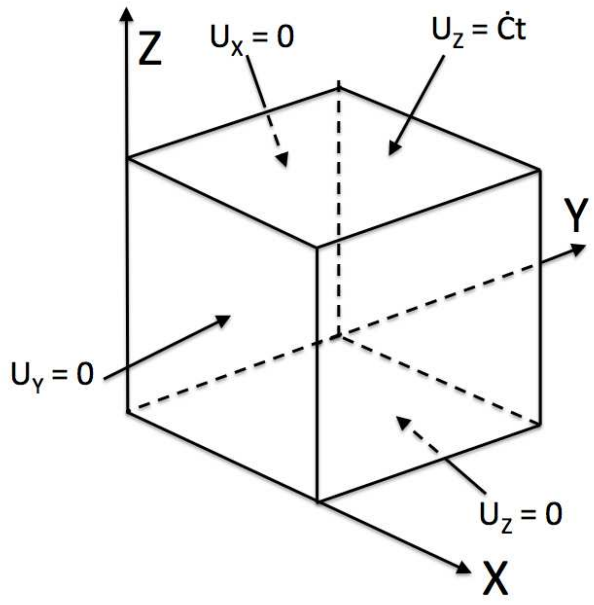

(b)

Figure 3: (a) The FE mesh and distribution of one of the Euler angles in the representative volume. (b) Boundary conditions to obtain uniaxial stress-strain response.

Most of the model parameters, apart from those involving defect terms, are first calibrated from the stress-strain curve of unirradiated pure-iron. In the CPFEM simulations, mobile and immobile density values of 40 and $30 \mu \mathrm{m}^{-2}$ are considered for pure-iron, respectively, based on a total density of $70 \pm 20 \mu \mathrm{m}^{-2}$ reported in [14]. The neutron irradiation of pureiron to 0.1 dpa results in the formation of small vacancy clusters and $1 / 2<111>$ SIA loops observed from TEM and PAS analyses [15]. However, the vacancy clusters provide negligible resistance to dislocation motion due to their small size as compared to the much larger SIA loops. This has also been shown using the Orowan's strengthening model in [15]. Hence, based on this observation, only the SIA loop density and mean size information reported in [15] together with the stress-strain curve provided in [14] is used to calibrate the SIA loop related model parameters. The calibrated parameter values are shown in Table.2 and a comparison of the stress-strain curves is shown in Fig.4. A comparison between Tables. 1 and 2 shows that a subset of the parameters required re-evaluation. Compared to DD, a significant lowering of dynamic recovery $\left(k_{d y n}\right)$, barrier strengths $\left(q_{p}, q_{i}\right)$ and loop annihilation $(\eta)$ related parameters is necessary to match the experimental curves. 
Table 2: Parameters of crystal plasticity model calibrated from tensile experiments on pure-iron [14]

\begin{tabular}{|c|c|c|c|c|c|}
\hline$k_{\text {mul }}$ & 0.28 & $k_{\text {dyn }}$ & 15 & $q_{p}$ & 0.07 \\
\hline$q_{i}$ & 0.046 & $\beta_{i}$ & 0.4 & $\eta$ & 5 \\
\hline
\end{tabular}

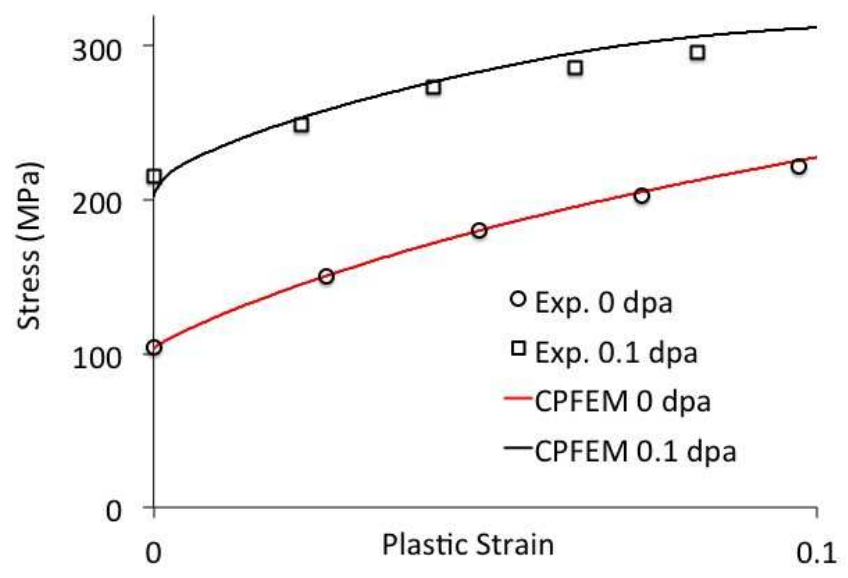

Figure 4: Comparison of stress-strain evolution between crystal plasticity finite element method (CPFEM) simulations and experiments for unirradiated and irradiated pure-iron. The experimental stress-plastic strain curve is reproduced from [14].

For the iron-copper alloys with $0.1 \%$ and $0.3 \%$ copper content, an increase in the postyield hardening is observed in [14]. This trend is non-reproducible by simply incorporating the increased initial dislocation densities of $90 \pm 20 \mu \mathrm{m}^{-2}$ for these two alloys from [14]. As discussed in [33], the increase in hardening can be due to a higher dislocation multiplication promoted by the solute atoms. Considering this mechanism, the $k_{m u l}$ parameter is recalibrated from the experimental stress-strain curves for these alloys. The pinning of dislocations caused slight increase of yield stress, which is captured by modifying the barrier strength of iron $0.3 \%$ copper alloy. The following parameters are recalibrated for the two alloys: $k_{m u l}=0.32$ for iron $0.1 \%$ copper, and, $k_{m u l}=0.32$ and $q_{p}=0.006$ for iron $0.3 \%$ copper. A comparison of the stress-strain curve with experiment is shown in Fig.5. In the CPFEM simulations, the mobile and immobile density values of 40 and $50 \mu^{-2}$ are considered, respectively, based on a total density of $90 \pm 20 \mu^{-2}$.

Under neutron irradiation to $0.1 \mathrm{dpa}$, small vacancy clusters, vacancy and copper clusters and SIA loops are observed in the iron-copper alloys, and are quantified using PAS, APT and TEM measurements in [15]. Similar to pure-iron, the small vacancy clusters provide negligible resistance, and the larger sized vacancy and copper clusters and SIA loops are the dominant barriers to dislocation motion [15]. Based on this observation, the crystal plasticity model parameters associated with the vacancy and copper clusters is calibrated using the experimental stress-strain curve for iron $0.1 \%$ copper provided in [14], and the cluster density and mean size information reported in [15]. With all other parameters remaining the same, $q_{c}=0.02$ provided a satisfactory agreement with experimental stress-strain curve and is shown in Fig. 6. 
The crystal plasticity model is subsequently validated for iron $0.3 \%$ copper using the defect density and mean size values provided in [15], and the corresponding stress-strain curve provided in [14]. A satisfactory agreement is obtained as can be observed from Fig.7. The defect density and mean size values obtained from [15] for pure-iron and iron-copper alloys under neutron-irradiation to $0.1 \mathrm{dpa}$ are listed in Table.3.

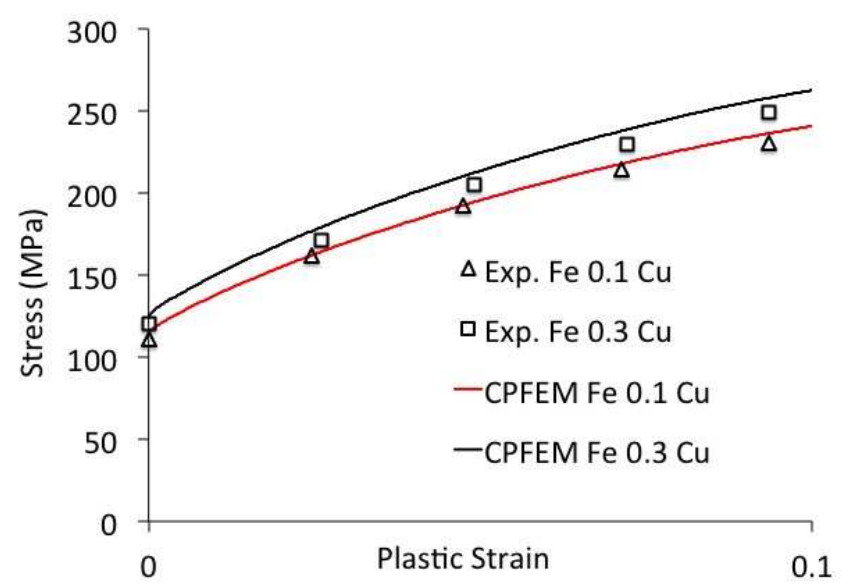

Figure 5: Comparison of stress-strain evolution between crystal plasticity finite element method (CPFEM) simulations and experiments for unirradiated iron-copper alloys. The experimental stress-plastic strain curve is reproduced from [14].

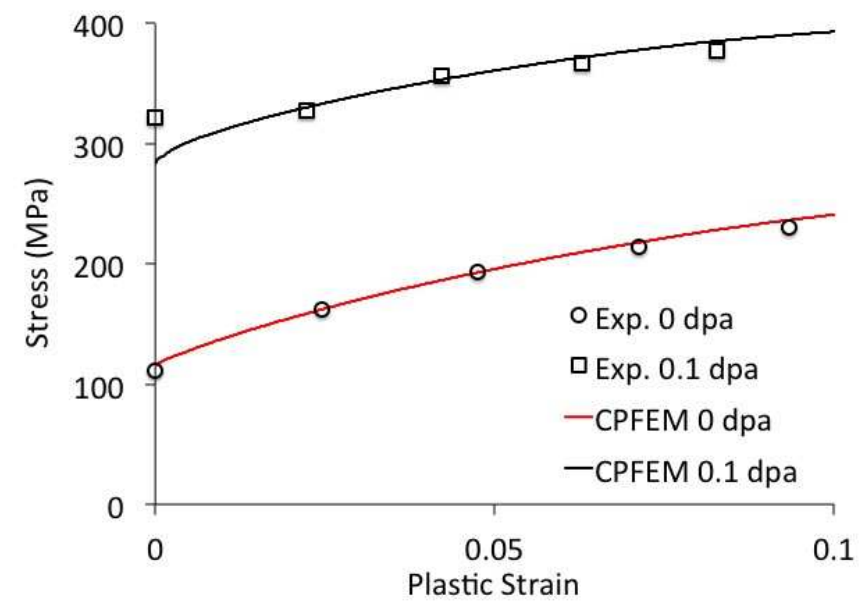

Figure 6: Comparison of stress-strain evolution between crystal plasticity finite element method (CPFEM) simulations and experiments for unirradiated and irradiated iron $0.1 \%$ copper alloy. The experimental stressplastic strain curve is reproduced from [14]. 
Table 3: Defect density $\left(10^{20} \mathrm{~m}^{-3}\right)$ and mean size $(\mathrm{nm})$ values obtained from [15] used for calibration and validation of the crystal plasticity model

\begin{tabular}{|c|c|c|c|c|}
\hline Alloy & Cluster Size & Cluster Density & Loop Size & Loop Density \\
\hline Pure Iron & 0 & 0 & 7 & 12.7 \\
\hline Iron $0.1 \% \mathrm{Cu}$ & 2.4 & 914 & 4.5 & 12.3 \\
\hline Iron $0.3 \% \mathrm{Cu}$ & 3 & 2177 & 5.2 & 35.5 \\
\hline
\end{tabular}

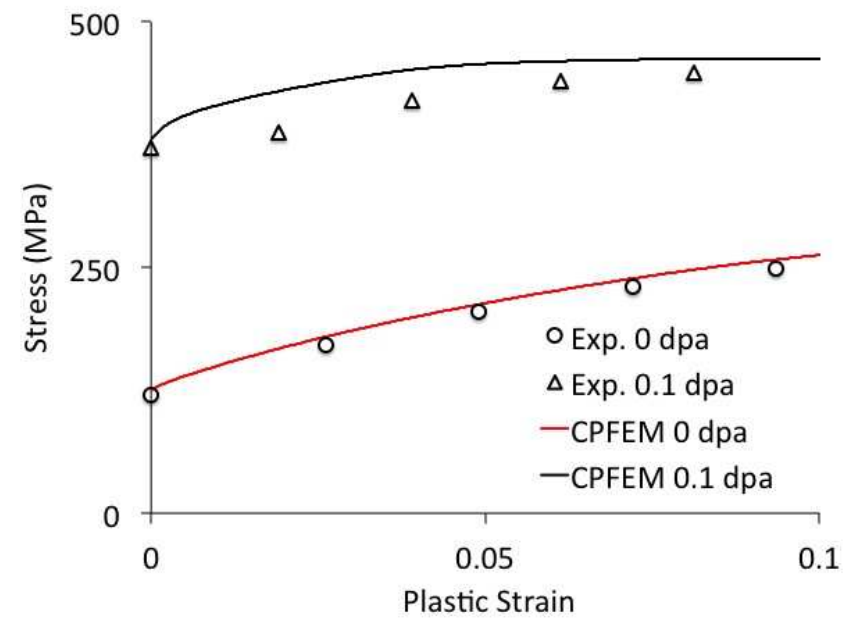

Figure 7: Comparison of stress-strain evolution between crystal plasticity finite element method (CPFEM) simulations and experiments for unirradiated and irradiated iron $0.3 \%$ copper alloy. The experimental stressplastic strain curve is reproduced from [14].

For the irradiated iron $0.3 \%$ copper alloy, the sensitivity of stress-strain evolution on the dislocation density and orientation distribution is further investigated. For the first case, the mobile and immobile dislocation densities are modified to 50 and $40 \mu^{-2}$, respectively, but the same random orientation of grains is maintained. For the second case, a different uniform random set of grain orientations is considered while the original mobile and immobile dislocation densities are utilized. The stress-strain evolution for these two cases is compared in Fig.8. As can be seen from the figure, a minor change in the stress-strain evolution occurs due to the variations in the mobile to immobile dislocation density ratio and orientation. This also shows that the number of grains considered in this study is reasonable to provide macroscopic flow-stress response from microstructure scale crystal plasticity simulations. 


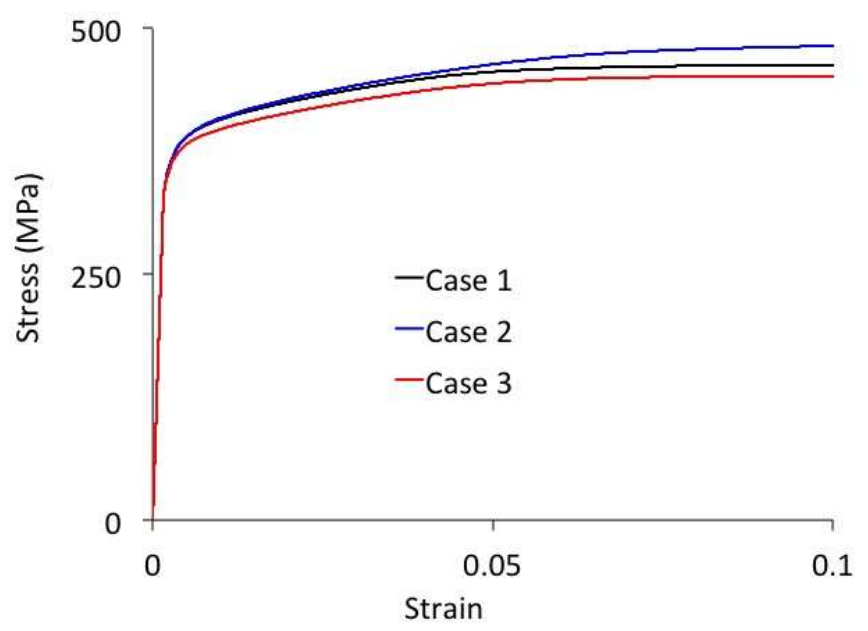

Figure 8: Sensitivity of stress-strain evolution on the dislocation density and orientation distribution for irradiated iron $0.3 \%$ copper alloy. Case 1: Original random orientation of grains with $\rho_{M}^{\alpha}=40$ and $\rho_{I}^{\alpha}=50$ $\mu \mathrm{m}^{-2}$; Case 2: Original random orientation of grains with $\rho_{M}^{\alpha}=50$ and $\rho_{I}^{\alpha}=40 \mu \mathrm{m}^{-2}$; Case 3: Different set of random grain orientation with $\rho_{M}^{\alpha}=40$ and $\rho_{I}^{\alpha}=50 \mu \mathrm{m}^{-2} \cdot \rho_{M}^{\alpha}$ and $\rho_{I}^{\alpha}$ are the mobile and immobile dislocation densities, respectively.

\subsection{Comparison between crystal plasticity and an analytical model of hardening}

The influence of irradiation induced defect on the increase of yield stress is typically modeled using the Orowan's strengthening model

$$
\Delta \sigma=\alpha M G b \sqrt{N d}
$$

where $\alpha$ is the strength factor that depends on the defect type and size [27,34], and, $\mathrm{M}$ is the Taylor factor. Eq.16 has been used in [15] to quantify the contribution of the different defect types such as clusters, precipitates and loops on the overall increase of yield-strength for a RPV steel and its model alloys irradiated to $0.1 \mathrm{dpa}$. From their analyses it was shown that the small vacancy clusters have negligible influence on hardening. A decrease in the strength factor value $(\alpha)$ with reduction in mean defect size was obtained from their calibration as shown in Fig.9. However, an opposite trend was obtained for the vacancy and copper clusters as can be seen from the figure. Further, from their analyses it was concluded that a quadratic superposition of the resistances provided a better correlation with experimental results.

In the crystal plasticity model the same experimental data-set is utilized [15] for pure-iron and iron-copper alloys. From a systematic calibration and validation process it is observed that constant barrier strength parameter $\left(q_{p}, q_{i}\right.$ and $\left.q_{c}\right)$ values is able to reproduce the experimental stress-strain response reasonably well within the range of mean defect size and density. This may be due to the consideration of a more detailed interaction behavior between dislocation and defects in the crystal plasticity framework. Moreover, a linear superposition of the resistances ( $r=1$ in Eq.6) is observed to be more suitable to model the increase of yield-strength.

These two models are further compared by utilizing the microstructural information provided in [16] for pure-iron and iron $0.1 \%$ copper alloy irradiated to different dose-levels. Since, the data provided for iron $0.3 \%$ copper alloy was incomplete, it could not be used in this comparative study. A comparison of the yield stress increase between the two models 
and experiments [15] is shown in Fig.10 and 11 for pure-iron and iron $0.1 \%$ copper alloy, respectively. For pure-iron irradiated to $0.05 \mathrm{dpa}$, both the models estimate a similar increase in yield strength (Fig.10(b)). At 0.2 dpa, both the defect density and mean size evolves significantly (Fig.10(a)) though the yield strength increase almost saturates (Fig.10(b)). However, by utilizing this defect information at $0.2 \mathrm{dpa}$ both the models predict a higher increase of yield strength as compared to experiments, though the crystal model is closer to the experimental values (Fig.10(b)).

For the iron $0.1 \%$ copper alloy, the SIA loop density and mean size increase with doselevel as can be observed in Fig. 11(a)), though much less severe than pure-iron. Also, the vacancy and copper cluster mean size shows very little increase with dose, while the number density is even observed to decrease at $0.2 \mathrm{dpa}$. Based on this defect information, a much closer estimate of yield-strength increase is predicted from the models (Fig.11(b)). However, a saturation of yield-strength increase as seen in the experiment is still not obtained from both the crystal plasticity and analytical model. The results for the iron $0.1 \%$ copper alloy suggests that the defect mean size and density values at 0.2 dpa provided in [16] may require re-evaluation.

The stress-strain and strain-hardening behavior as obtained from the crystal plasticity model is compared in Fig.12. An insignificant change in the strain-hardening rate is observed till 0.025 engineering strain for all the dose-levels. Beyond this strain-level a reduction in the strain-hardening rate is noticeable for 0.1 and $0.2 \mathrm{dpa}$, and is due to the increased annihilation of the SIA loops by the dislocations. For pure-iron irradiated to 0.2 dpa, a strain softening behavior can even be observed beyond 0.025 engineering strain. At strains lower than 0.025, a small increase in the strain-hardening rate can be observed for all the dose-levels. This increase can be attributed to an increased trapping of dislocations by the SIA loops. A similar trend can be observed from Fig. 13 in iron $0.1 \%$ copper alloy as well. For this alloy, the reduction in the strain-hardening rate is however lower than pure-iron since the primary softening mechanism is attributed to dislocation-SIA loop interaction in the model.

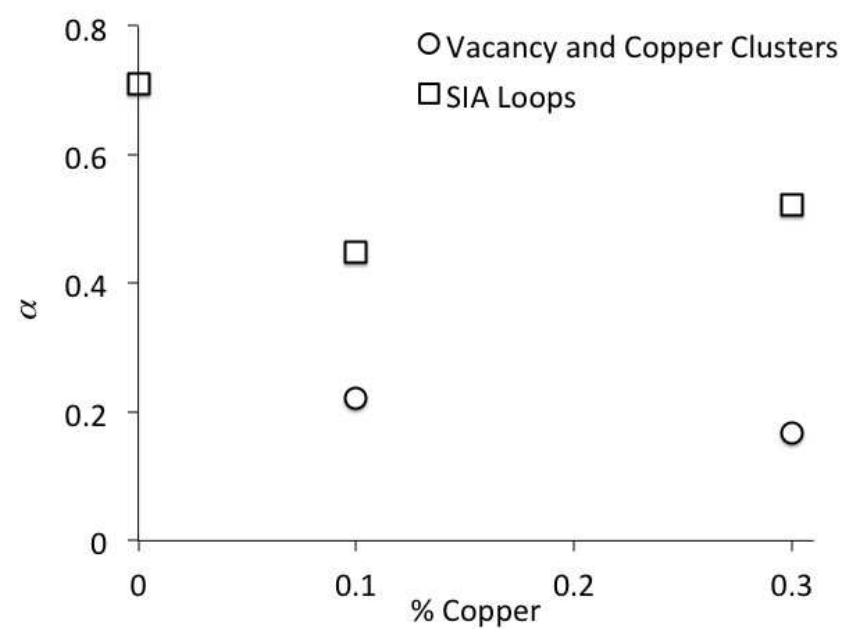

Figure 9: The variation of SIA loop and vacancy and copper cluster strengths ( $\alpha$ in Eq.16) for pure-iron and iron-copper alloys. The strength factor values are reproduced from [15]. 


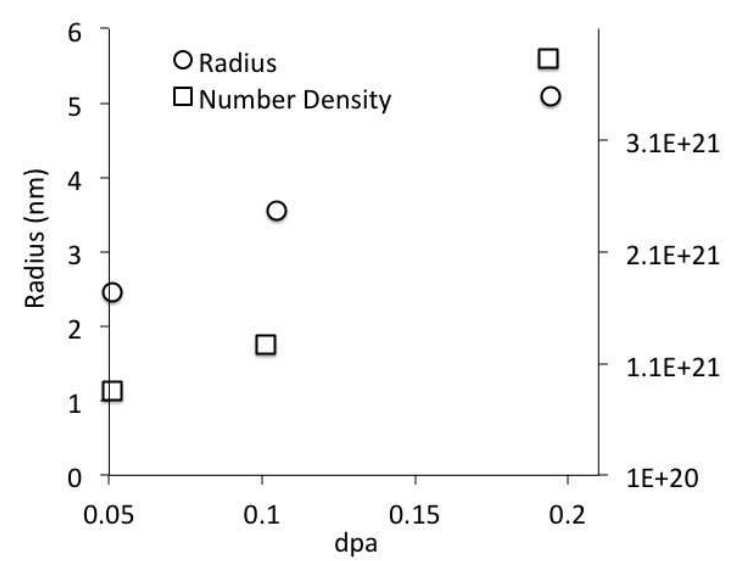

(a)

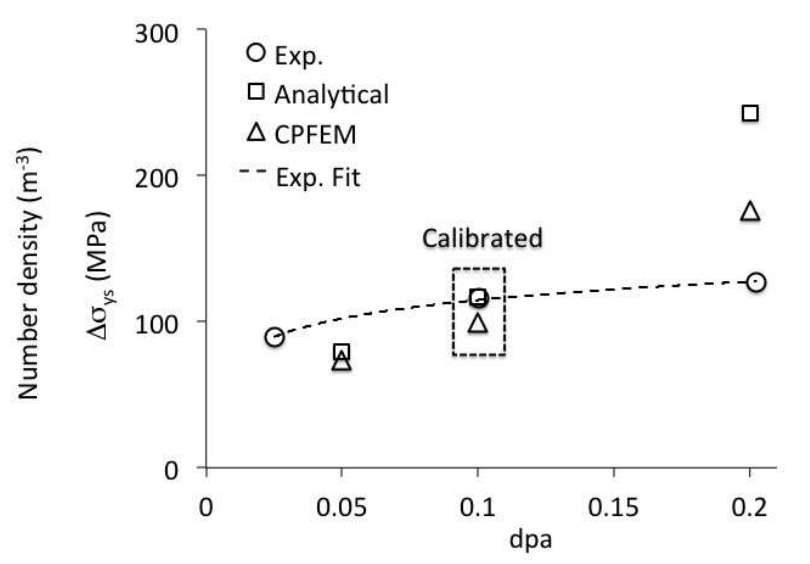

(b)

Figure 10: (a) Mean size and number density of SIA loops in pure-iron irradiated to different dose levels reproduced from [16]. (b) Comparison of increase in yield stress $\left(\Delta \sigma_{y s}\right)$ obtained from crystal plasticity and analytical model.

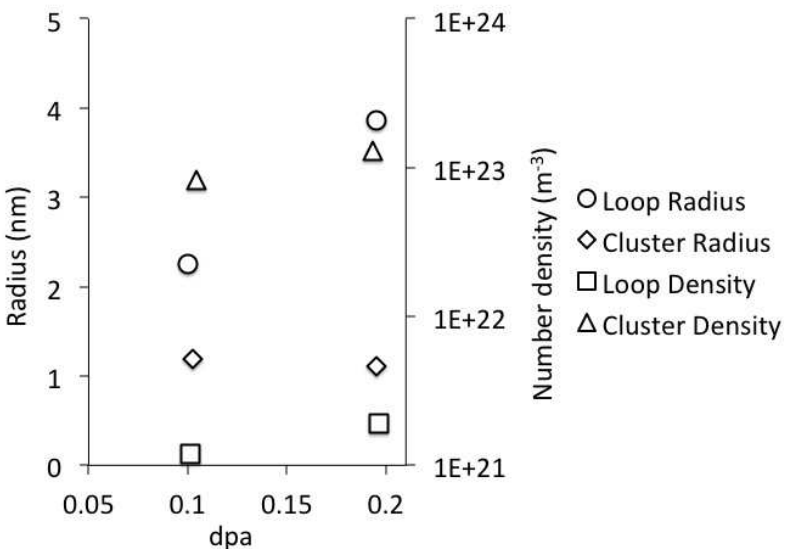

(a)

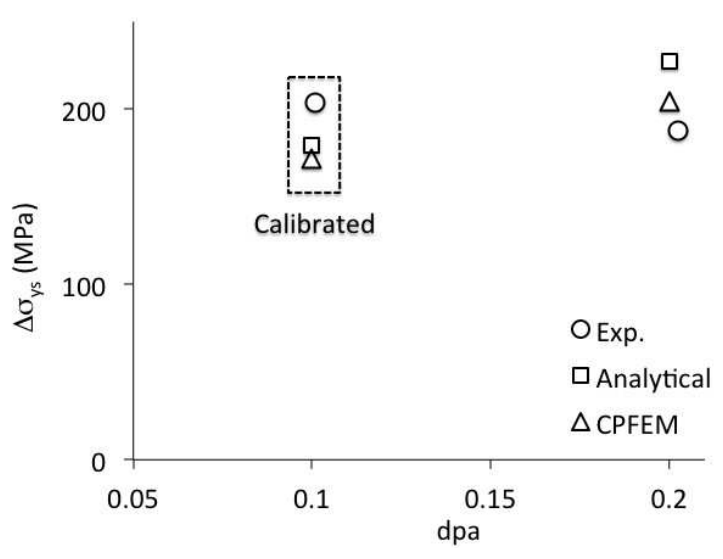

(b)

Figure 11: (a) Mean size and number density of SIA loops in pure-iron irradiated to different dose levels reproduced from [16]. (b) Comparison of increase in yield stress $\left(\Delta \sigma_{y s}\right)$ obtained from crystal plasticity and analytical model. 


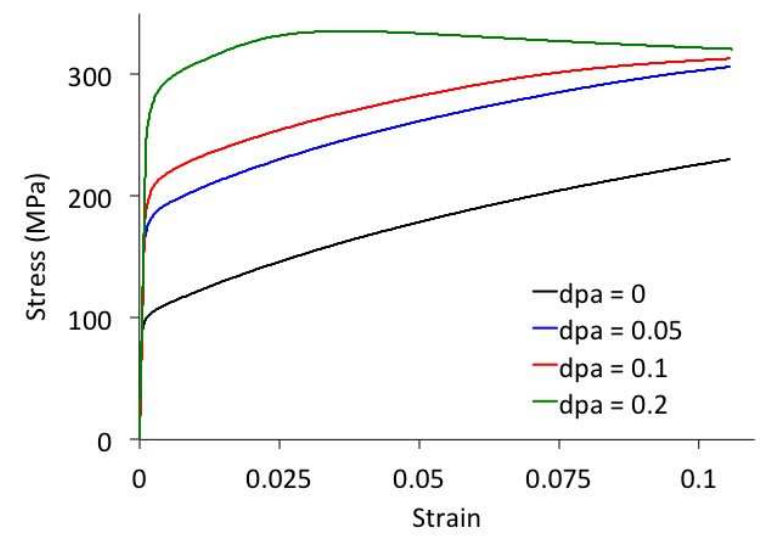

(a)

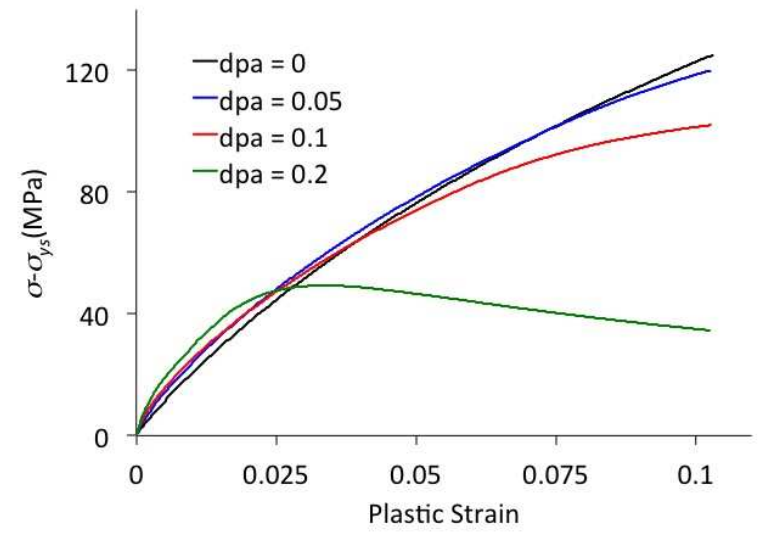

(b)

Figure 12: Comparison of (a) stress-strain, and, (b) strain-hardening, evolution for pure-iron irradiated to different dose-levels obtained from crystal plasticity model.

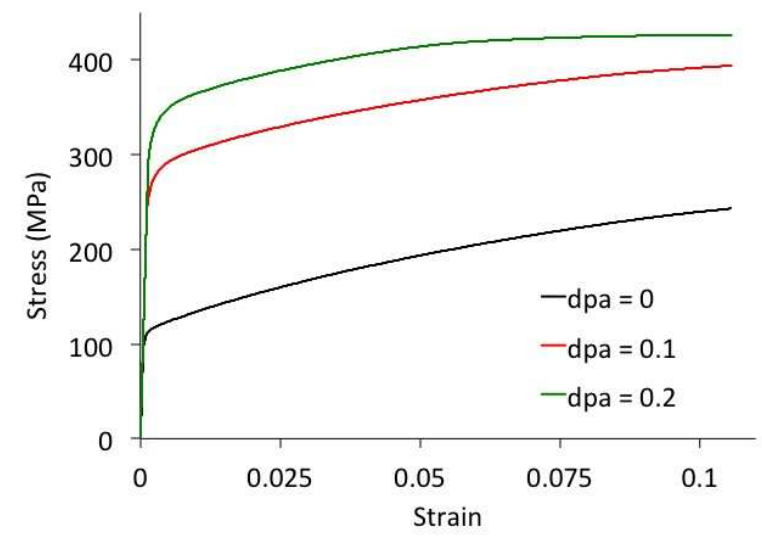

(a)

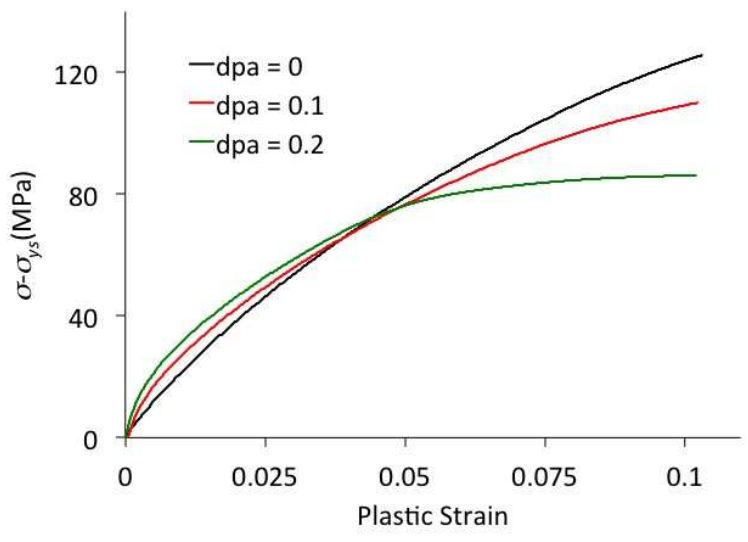

(b)

Figure 13: Comparison of (a) stress-strain, and, (b) strain-hardening, evolution for iron $0.1 \%$ copper irradiated to different dose-levels obtained from crystal plasticity model.

\section{Conclusion}

A crystal plasticity model has been developed in this work to capture the interaction of dislocations with SIA loops and vacancy and copper clusters formed due to neutronirradiation of pure-iron and iron-copper alloys. The model considers the initial pinning of the mobile dislocations by the loops and the anisotropic anhihilation of the loops once the dislocations overcome the barrier to glide. For the vacancy and copper clusters, an isotropic resistance model similar to Orowan's strengthening model has been used.

A satisfactory agreement of the stress-plastic strain evolution of the model could be obtained with dislocation dynamics (DD) simulations with minimum parameterization. Subsequently, the experimental data of stress-strain, and yield strength increase, in conjunction with defect density and mean size has been used to obtain the flow stress behavior in pure-iron and iron-copper alloys. The calibration and validation procedure involved crystal plasticity 
finite element method (CPFEM) simulations of a polycrystalline representative volume with random grain orientation to obtain the average stress-strain response, which has been then compared with experimental curves. Reduction of some of the parameters calibrated from the DD simulations was necessary to match the experimental curves. From the systematic calibration procedure, the different model parameters could be separately obtained that were then satisfactorily validated with the irradiated iron $0.3 \%$ copper alloy. From the results, it can be concluded that a constant set of parameters can provide reasonable comparison with experimental stress-strain response within the dpa range considered. In the model, only the mean size of the defects have been considered due to the absence of size distribution information. Moreover, a mean dislocation density has been assumed in all the grains, though in reality spatial variations will exist. Consideration of these factors can have some influence on the average response obtained from this work and requires further investigation.

The increase in yield-strength for pure-iron and iron-copper alloys at different dose-levels has then been investigated using the crystal plasticity model. For pure-iron a saturation of yield-strength increase, as observed experimentally, could not be obtained from this model. On the contrary, a near-linear increase could be observed similar to the analytical model using Orowan's strength equation with suitably calibrated parameters. However, for the irradiated iron $0.1 \%$ copper alloy, a much better agreement of the yield-strength increase could be obtained from the crystal plasticity model. However, for both the materials, the Orowan's strengthening model over estimates the yield strength increase as compared to the crystal plasticity model. The strain-hardening rate at different dose-levels has also been compared for pure-iron and iron $0.1 \%$ copper alloy. At 0.2 dpa and beyond $2.5 \%$ strain, strain softening and near-zero strain-hardening has been observed for pure-iron and iron $0.1 \%$ copper alloy, respectively.

\section{Acknowledgment}

This work was funded by the DOE Light Water Reactor Sustainability Program. This manuscript was authored by contractors (Battelle Energy Alliance, LLC) of the US Government under the Department of Energy Contract No DE-AC07-05ID14517. Accordingly, the publisher by accepting the paper for publication acknowledges that the US Government retains a nonexclusive, paid-up, irrevocable, world-wide license to publish or reproduce the published form of this manuscript, or allow others to do so, for US Government purposes.

[1] C. English, J. Hyde, Radiation Damage of Reactor Pressure Vessel Steels, volume 4, Elsevier, 2012, pp. 151-180.

[2] E. Eason, G. R. Odette, R. Nanstad, T. Yamamoto, A physically based correlation of irradiation-induced transition temperature shifts for RPV steels, Journal of Nuclear Materials 433 (2013) 240-254.

[3] Editorial, Perfect - prediction of irradiation damage effects on reactor components: A summary, Journal of Nuclear Materials 406 (2010) 2-6.

[4] A. L. Gurson, Continuum theory of ductile rupture by void nucleation and growth: Part I-yield criteria and flow rules for porous ductile media, Journal of Engineering Materials 99 (1997) 2-15. 
[5] G. Rousselier, Ductile fracture models and their potential in local approach of fracture, Nuclear Engineering and Design 105 (1987) 97-111.

[6] F. Beremin, A local criterion for cleavage fracture of a nuclear pressure vessel steel, Metals and Materials Transaction A 14A (1983) 2277-2287.

[7] W. Weibull, A statistical distribution function of wide applicability, Journal of Applied Mechanics 18 (1953) 293-297.

[8] B. Tanguy, J. Besson, R. Piques, A. Pineau, Ductile to brittle transition of an A508 steel characterized by Charpy impact test. Part II: modeling of the Charpy transition curve, Engineering Fracture Mechanics 72 (2005) 413-434.

[9] B. Tanguy, C. Bouchet, S. Buget, J. Besson, Local approach to fracture based prediction of the $\Delta T_{56 J}$ and $\Delta T_{K I C, 100}$ shifts due to irradiation for an a508 pressure vessel steel, Engineering Fracture Mechanics 73 (2006).

[10] M. K. Samal, M. Seidenfuss, E. Roos, B. K. Dutta, H. S. Kushwaha, Experimental and numerical investigation of ductile-to-brittle transition in a pressure vessel steel, Material Science and Engineering A 496 (2008) 25-35.

[11] P. Chakraborty, S. B. Biner, A unified cohesive zone approach to model the ductile to brittle transition of fracture toughness in reactor pressure vessel steels, Engineering Fracture Mechanics 131 (2014) 194-209.

[12] P. Chakraborty, S. B. Biner, Parametric study of irradiation effects on the ductile damage and flow stress behavior in ferritic-martensitic steels, Journal of Nuclear Materials 465 (2015) 89-96.

[13] G. R. Odette, M. Y. He, T. Yamamoto, On the relation between irradiation induced changes in the master curve reference temperature shift and changes in strain hardened flow stress, Journal of Nuclear Materials 367-370 (2007) 561-567.

[14] M. Lambrecht, L. Malerba, A. Almazouzi, Influence of different checmical elements on irradiation-induced hardening embrittlement of RPV steels, Journal of Nuclear Materials 378 (2008) 282-290.

[15] M. Lambrecht, E. Meslin, L. Malerba, M. Hernandez-Mayoral, F. Bergner, P. Pariege, B. Radiguet, A. Almazouzi, On the correlation between irradiation-induced microstructural features and the hardening of reactor pressure vessel steels, Journal of Nuclear Materials 406 (2010) 84-89.

[16] E. Meslin, M. Lambrecht, M. Hernandez-Mayoral, F. Bergner, L. Malerba, P. Pariege, B. Radiguet, A. Barbu, D. Gomez-Briceno, A. Ulbricht, A. Almazouzi, Characterization of neutron-irradiated ferritic model alloys and a rpv steel from combined APT, SANS, TEM and PAS analyses, Journal of Nuclear Materials 406 (2010) 73-83. 
[17] A. Arsenlis, M. Rhee, G. Hommes, R. Cook, J. Martin, A dislocation dynamics study of the transition from homogeneous to heterogeneous deformation in irradiated bodycentered cubic iron, Acta Materialia 60 (2012) 3748-3757.

[18] F. Roters, P. Eisenlohr, L. Hantcherli, D. D. Tjahjanto, T. R. Bieler, D. Raabe, Overview of constitutive laws, kinematics, homogenization and multiscale methods in crystal plasticity finite-element modeling: Theory, experiments, applications, Acta Materialia 58 (2010) 1152-1211.

[19] R. Lagneborg, B. H. Forsen, A model based on dislocation distributions for workhardening and the density of mobile and immobile dislocations during plastic flow, Acta Metallurgica 21 (1973) 781-790.

[20] A. Ma, F. Roters, A constitutive model for fcc single crystals based on dislocation densities and its application to uniaxial compression of aluminium single crystals, Acta Materialia 52 (2004) 3603-3612.

[21] A. Patra, D. L. McDowell, Continuum modeling of localized deformation in irradiated bcc materials, Journal of Nuclear Materials 432 (2013) 414-427.

[22] U. F. Kocks, A statistical theory of flow stress and work-hardening, Philosophical Magazine 13 (1966) 541-566.

[23] Y. Estrin, Unified Constitutive Laws of Plastic Deformation, Academic Press, New York, USA, 1996.

[24] M. B. P. Franciosi, A. Zaoui, Latent hardening in copper and aluminium single crystals, Acta Metallurgica 28 (1980) 273-283.

[25] N. R. Barton, A. Arsenlis, J. Marian, A polycrystal plasticity model of strain localization in irradiated iron, Journal of the Mechanics and Physics of Solids 61 (2013) 341-351.

[26] S. J. Zinkle, B. N. Singh, Microstructure of neutron-irradiated iron before and after tensile deformation, Journal of Nuclear Materials 351 (2006) 269-284.

[27] S. Kotrechko, V. Dubinko, N. Stetsenko, D. Terentyev, X. He, M. Sorokin, Temperature dependence of irradiation hardening due to dislocation loops and precipitates in RPV steels and model alloys, Journal of Nuclear Materials 464 (2015) 6-15.

[28] C. Kohler, P. Kizler, S. Schmauder, Atomistic simulation of precipitation hardening in $\alpha$ iron: influence of precipitate shape and chemical composition, Modelling and Simulation in Materials Science and Engineering 13 (2005) 35-45.

[29] D. Gaston, C. Newman, G. Hansen, D. Lebrun-Grandie, Moose: A parallel computational framework for coupled systems of nonlinear equation, Nuclear Engineering and Design 239 (2009) 1768-1778.

[30] S. Balasubramanian, Polycrystalline Plasticity: Application to Deformation Processing of Lightweight Metals, Ph.D. thesis, Massachusetts Institute of Technology, 1998. 
[31] P. Chakraborty, S. B. Biner, Y. Zhang, B. Spencer, Crystal plasticity model of reactor pressure vessel embrittlement in Grizzly, Technical Report INL/EXT-15-34786, Idaho National Laboratory, 2015.

[32] D. Deka, D. S. Joseph, S. Ghosh, M. J. Mills, Crystal plasticity modeling of deformation and creep in polycrystalline Ti-6242, Metallurgical and Materials Transactions A 37A (2006) 1371-1388.

[33] C. G. Schmidt, A. K. Miller, The effect of solutes on the strength and strain hardening behavior of alloys, Acta Metallurgica 30 (1982) 615-625.

[34] X. Hu, D. Xau, T. S. Byun, B. D. Wirth, Modeling of irradiation hardening of iron after low-dose and low-temperature neutron irradiation, Modelling and Simulation in Materials Science and Engineering 22 (2014) 065002. 